\title{
Experiments with the TER Tele-echography Robot
}

\author{
Adriana Vilchis ${ }^{1}$, Jocelyne Troccaz ${ }^{1}$, Philippe Cinquin ${ }^{1}$, Agnes Guerraz ${ }^{2}$, \\ Franck Pellisier ${ }^{2}$, Pierre Thorel ${ }^{2}$, Bertrand Tondu ${ }^{3}$, Fabien Courrèges ${ }^{4}$, \\ Gérard Poisson ${ }^{4}$, Marc Althuser ${ }^{5}$, and Jean-Marc Ayoubi ${ }^{5}$ \\ ${ }^{1}$ TIMC/IMAG laboratory, Domaine de la Merci, F-38706 La Tronche cedex - France \\ \{adriana.vilchis, jocelyne.troccaz\}@imag.fr \\ ${ }^{2}$ France Telecom R\&D \\ ${ }^{3}$ INSA Toulouse \\ ${ }^{4}$ LVR Bourges \\ ${ }^{5}$ CHU Grenoble
}

\begin{abstract}
This paper presents a master-slave system applied to the remote diagnosis from echographic data. The motion of the master manipulator is remotely controlled by a physician and reproduced by a slave robot carrying the echographic probe. The contact force between the probe and the patient is fed back to operator allowing him to have a haptic virtual environment. The innovation of this haptic control is to preserve medical expert propioception and gesture feelings, which are necessary to synchronize the ultrasound images with the motion made by the medical doctor. The slave robot is a cable-driven manipulator using pneumatic artificial muscle actuators to control the motion of the ultrasound probe. In this paper we present the architecture and the performances of the slave robot and the first experiments of the master-slave remote echography system for examinations of pregnant women.
\end{abstract}

\section{Introduction}

Among many types of medical equipment, ultrasound diagnostic systems are widely used because of their convenience and innocuously. Performing an ultrasound examination involves good hand-eye coordination and the ability to integrate the acquired information over time and space; the physician has to be able to mentally build 3D information from both the 2D images and the gesture information and to put a diagnosis from this information. Some of these specialized skills may lack in some healthcare centers or for emergency situations. Tele-consultation is therefore an interesting alternative to conventional care. Development of a high performance remote diagnostic system, which enables an expert operator at the hospital to examine a patient at home, in an emergency vehicle or in a remote clinic, may have a very significant added value.

Some previous tele-echography and robot-based echography systems have been developed [1-8]. Some of these approaches are purely tele-medicine projects (see [5] for example). A second class of systems allows to automate an echographic examination using a robot $[3,4]$. Finally, a last category of robot-based systems enables the remote examination of patients by a distant expert with $[3,6]$ or without $[2,8]$ force feedback. Many of the robot-based systems integrate conventional robot architectures 
and actuation. One objective of this research was to propose a new architecture of low weight, compliant and portable medical robots.

\section{Overview of the TER System}

\subsection{Description of the TER System}

The tele-operated TER system allows the expert physician to move by hand a virtual probe in a natural and unconstrained way and safely reproduces this motion on the distant robotic site where the patient is. For a global description of the TER project see [9]. Figure 1.a shows a schematic diagram of the TER system. The physician located in the master site moves the virtual probe placed on a haptic device to control the real echographic probe placed on the slave robot. The haptic control station in the master site is developed to give a realistic environment of what remotely occurs [10]. It integrates a PHANToM device (from SensAble Device Inc) which has 6 degrees of freedom (dof) and renders 3D-force information. Position and orientation tracking of the virtual probe is performed within a workspace of $16 \mathrm{~cm} \times 13 \mathrm{~cm} \times 13 \mathrm{~cm}$ and with maximum a force of $6.4 \mathrm{~N}$. Real time force feedback information and a virtual geometric model of the patient (see [11]) are rendered to the expert operator. The obtained ultrasound image is continuously sent from the slave site to the operator that has to perform the examination and to provide a diagnosis. The precise position of the ultrasound probe is provided by an optical localizer and is sent to the master site where the position is represented as a visual information. A non-expert operator is located close to the patient and supervises the procedure that he can interrupt in an emergency case. The patient can at all times communicate with the operator or with the expert. Two IDSN $128 \mathrm{~kb} / \mathrm{s}$ connections are used; one is for the Visio-phonic data and echographic images and the other one is for the transmission of the control information for the slave-robot.

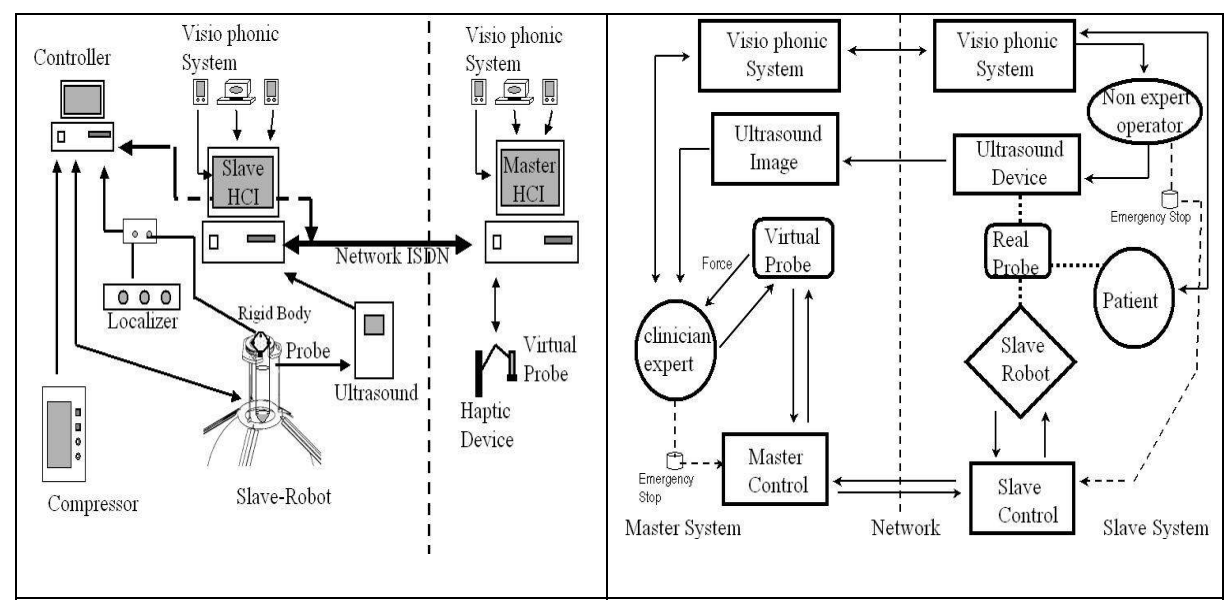

Fig. 1. Diagram of the TER system. (a) Schematic diagram. (b) Functional diagram 


\subsection{Procedure of Tele-echographic Robotic Examination}

The procedure of the tele-echographic robotic examination is performed in three phases: initialization, echographic examination and end of the session. Table 1 shows the steps of a typical procedure with the TER system and figure 1.b gives its functional diagram. Medical experts who tested the system followed this procedure.

Table 1. Procedure of examination of TER system

\begin{tabular}{|c|c|}
\hline Master site & Slave site \\
\hline $\begin{array}{l}\text { - Initialization of: } \\
\text { - Virtual Probe parameters } \\
\text { - Communication parameters } \\
\text { - Visio phonic parameters } \\
\text { - Haptic Environment } \\
\text { - Establishing communications } \\
\text { - Examination (continuous loop until end of } \\
\text { session): } \\
\text { - Motion of the Virtual Probe } \\
\text { - Analysis of ultrasound images } \\
\text { - Ask for the tuning of image parame- } \\
\text { ters of the echographic system } \\
\text { - Communication with the patient } \\
\text { or with the non expert operator } \\
\text { - End of session: } \\
\text { - Disconnection with the slave robot }\end{array}$ & $\begin{array}{l}\text { - Position of the slave robot relatively to the } \\
\text { patient body } \\
\text { - Gel application } \\
\text { - Initialization of: } \\
\text { - Robot parameters } \\
\text { - Communication parameters } \\
\text { - Visio phonic parameters } \\
\text { - Initial Position of Robot } \\
\text { - Waiting for communications } \\
\text { - Examination (continuous loop until end of } \\
\text { session): } \\
\text { - Motion of the slave robot } \\
\text { - Sending ultrasound images } \\
\text { - Tuning of image acquisition parameters } \\
\text { - by the non expert operator } \\
\text { - Communication with the medical expert } \\
\text { - End of session: } \\
\text { - Disconnection with the master haptic } \\
\text { - } \text { environment } \\
\text { - } \text { pake the slave robot away from the } \\
\text { - tor dinal discussion with the medical doc- }\end{array}$ \\
\hline
\end{tabular}

\subsection{Analysis of the Echographic Probe Motions}

To determine the motion and force range of the remote echography system, the executed motions and applied forces of a real echographic probe were analyzed while a physician was examining a patient with a conventional procedure. The observed motions and gestures zones were used to define the working surface used during the examination by the physician. In the application to the diagnosis of pregnant women, this surface depends on the stage of pregnancy. As the pregnancy evolves the working surface becomes larger and rounder. This surface is taken into account at the beginning of the examination. 
The condition of permanent contact of the echographic probe with the patient's body must be satisfied at each moment to allow the formation of images. The measurements of the effort variation on the abdomen during the examination using a dynamometer are of three types: minimal pressures from 0.6 to $0.7 \mathrm{dN}$, standard pressures from 0.8 to $1.1 \mathrm{dN}$ and maximum pressures from 1.3 to $1.5 \mathrm{dN}$. These measurements determine a range of authorized efforts, which have to be considered for remote examination.

\section{Ultrasound Slave-Robot Design}

\subsection{Slave Robot Mechanical Architecture}

The robot architecture is rather different from classical existing ones. The application simulation (a), the translation component of the slave robot prototype (b) and a close up of the orientation component of our current slave robot prototype attached to an abdomen phantom (c) are pictured in figure 2. The slave robot is a parallel uncoupled one. It includes two independent parallel structures having two independent groups of pneumatic artificial muscle actuators (McKibben artificial muscles) [12]. Thin and flexible cables are used to position and orient the echographic probe. The cables are connected to the pneumatic artificial muscles.

The artificial muscle is made of a thin rubber tube covered with a shell braided according to helical weaving. The muscle is closed by two ends, one being the air input and the other the force attachment point. When the inner tube is pressurized to a given pressure, the textile shell converts circumferential pressure force into axial contraction force, which reduces the muscle length while its radius increases. This means of actuation is simple, lightweight and has the added benefit of passive compliance. Moreover compressed air sources can be very easily found in the clinical environment.

The translation movements are controlled by four identical antagonistic artificial muscles connected by cables to a metal ring supporting the second parallel structure (see figure 2.b). The second parallel structure enables 3D orientation and translation along the $\mathrm{Z}$ axis of the echographic probe (see figure 2.c). It is actuated by four artificial muscles: three of them are identical and they control two of the three orientations and the translation. The fourth muscle controls the rotation of the ultrasound around its vertical axis. Both subsystems can be controlled simultaneously.

\section{Experimental Results}

\subsection{Performances of the Slave Robot}

Several control modes [13] can be used for such a robot: closed-loop pressure control, open-loop position control, closed-loop position control and hybrid position/force control. The results presented in this work correspond to the position openloop control (see picture 3). 

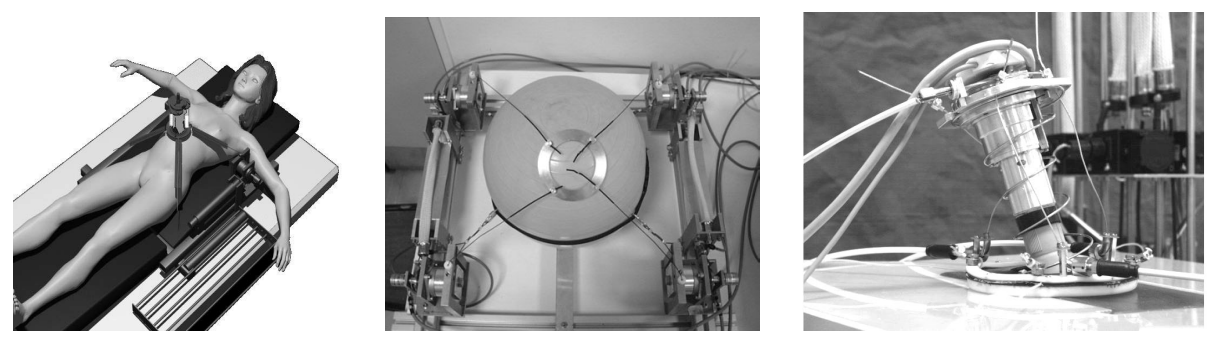

Fig. 2. Slave Robot. (a) Conception Design. (b) Translation Robot. (c) Orientation Robot

The data set of points $\left(\mathrm{P}_{\mathrm{d}}\right)$ generated by the motion of the virtual probe held by the expert are sent every 500ms to the robot controller which converts this information to cable lengths $\left(\mathrm{q}^{d}\right)$ using an inverse model of the robot. These lengths are turned to pressure joint values $(\mathrm{Pi})$ to be sent to the muscles. PIDA and PID regulators were respectively implemented for the closed-loop joint control of the translation and orientation structures of the robot [14]. As compared to a conventional robot, using only an open-loop position control is compensated here by the fact that the operator closes the loop from the image interpretation. However, the model allowing to compute cable lengths from Cartesian orders is not very precise because the working surface is a free-form surface and is not modeled perfectly for each patient.

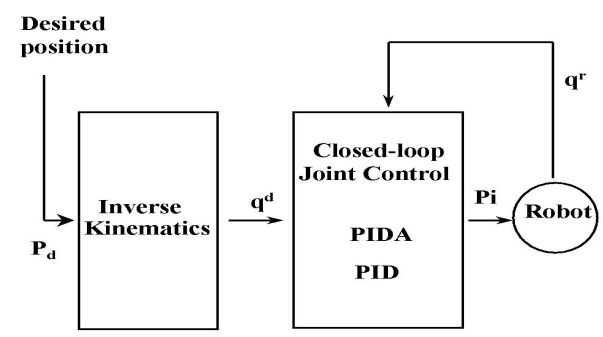

Fig. 3. Position Open-loop Control

The muscles have an intrinsic response time of $340 \mathrm{~ms}$ due to friction and inertial effects. Figure 4 shows the joint errors for both structures of the slave robot for a single position. The error between 0 and $400 \mathrm{~ms}$ in the figure 4 corresponds to the error resulting fromr the previous position. In figure 4.a we can see that the joint errors for the translation joints converge to zero after $600 \mathrm{~ms}$, which represents a good response time. Figure 4.b shows that the orientation joint errors correctly converge to zero after $1000 \mathrm{~ms}$.

Figure 5 shows the position error during a tracking task. The desired "trajectories" are a circle and a straight line that are defined by a sequence of points on the working surface. The robot does not perform a perfect tracking of the trajectory. The behavior in the lower part of figure 5.a can be explained by the imprecision with the two channels of the input board that read the values of the encoders associated with this part of the robot. In the example, the distance of the initial point to the trajectory also con- 
tributes to a tracking error since a new order is sent before the previous position is reached. Finally, the error is also due to the fact that the control is independent for each muscle. It could be reduced with a controller providing global and asymptotic error convergence for the closed-loop control. In Figure 5 we can see that the robot is more precisely in the straight-line trajectory that in the circle trajectory.
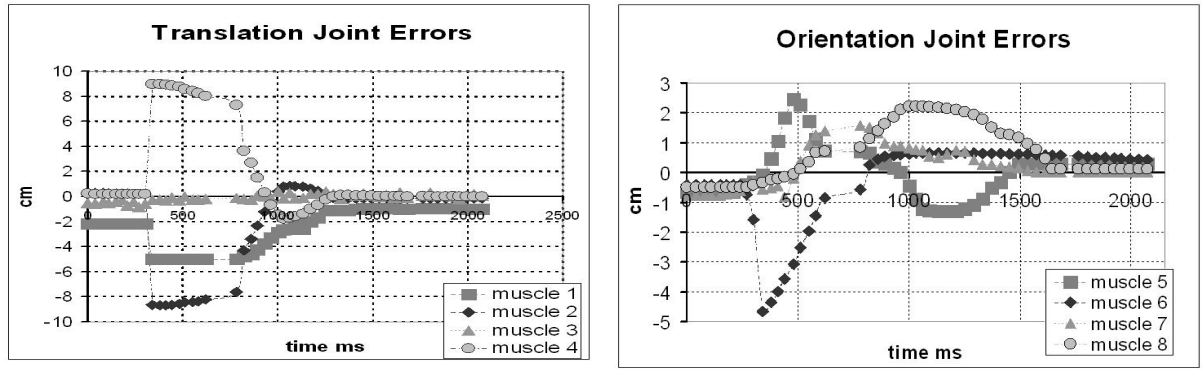

Fig. 4. Joints Errors. (a) Translation Joint Errors. (b) Orientation Joints Errors
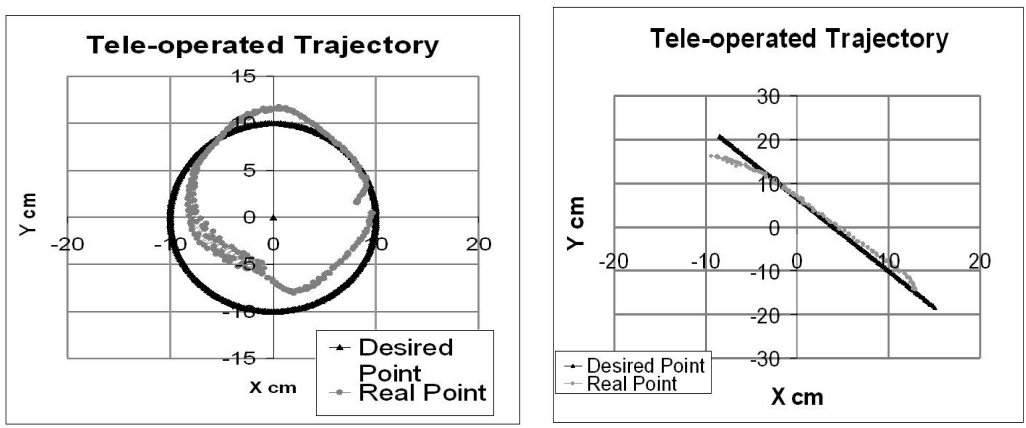

Fig. 5. Trajectory Errors. a) Circle Trajectory. b) Straight Line Trajectory

\subsection{Tele-operation Experiments}

After the integration of the system TER last january, some experiments have been performed using an echographic phantom (see figure 6.a) and one experiment in a voluntary patient. The objective of the test with the echographic phantom consisted in identifying the internal structures and in following them like in a real examination. The phantom was unknown to the medical experts. The objectives of the test performed with in a voluntary person belonging to the TER consortium patient consisted in identifying the internal organs and validating the ability of the robot TER to move on an unknown surface.

Three physicians (two obstetric gynecologists and one radiologist, all three being expert in echographic examinations) have carried out the examination with the echographic phantom and only one of the physicians has carried out the examination with 
the voluntary person. The medical experts handle the virtual probe fixed to the haptic system from the haptic information and from what that they see in the transmitted echographic images. Trajectory motions performed by the virtual probe are converted in a sequence of points, which are sent every $500 \mathrm{~ms}$ to the slave site. The system converts these points into joint values for the slave robot. The master and slave sites where about 10 kilometers distant. After experimenting with the system each physicians had to fill a questionnaire. The general appreciation of the system is good. The experiment time was between 45 minutes and 60 minutes approximately. Figure 6.b shows one of the echographic images seen by the experts during the experiments. Figure 7 presents different steps of experiment with the echographic phantom and figure 8 presents the experiment with a volunter.
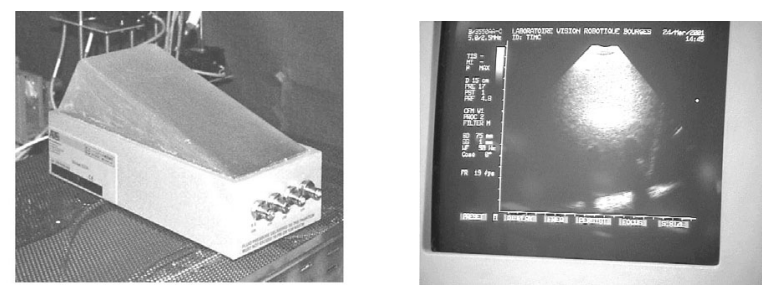

Fig. 6. (a) Echographic phantom used by the first experiments, (b) Echographic image of the internal structures of the phantom

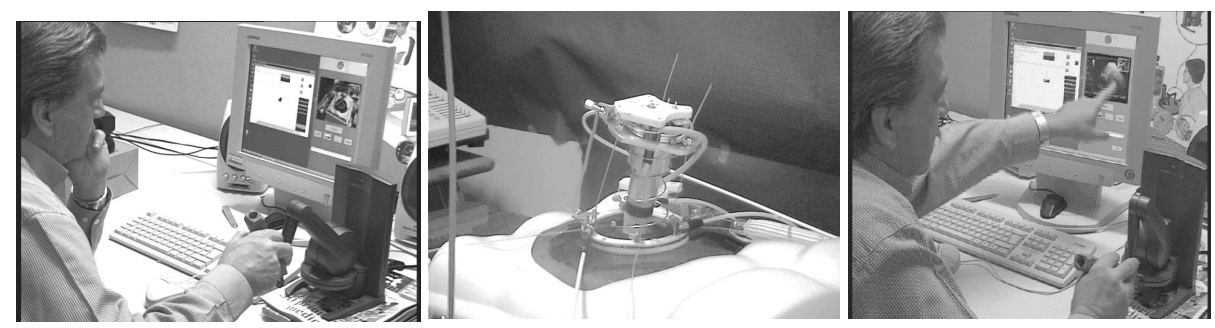

Fig. 7. (a) Doctor and haptic device (Master site), (b) Slave Robot on a medical phantom, (c) Doctor recognizing internal structures

\section{Conclusion}

In this paper, an original slave robot architecture was described and preliminary experiments with the TER system were presented. The system enables a medical expert to perform in real time, via ISDN communication links, an echographic examination on a patient remotely located. The first experiments realized with the TER system are very promising.

The TER system was developed for the realization of pregnancy echographic examination but the system can naturally be used for other types of echographic examination like heart, abdomen and others. 

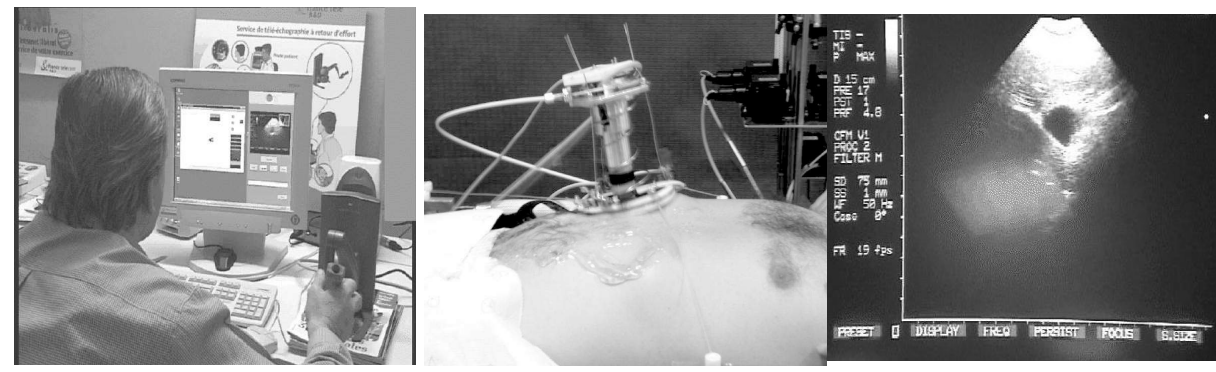

Fig. 8. (a) Doctor and the haptic device (Master site), (b) Slave Robot on voluntary patient, (c) Ultrasound Image seen by the doctor

Experiments demonstrated that the use of the TER system is very intuitive; there is a short adaptation time of 5 to 10 minutes necessary to the medical expert to feel comfortable with moving the virtual probe using the haptic device. The quality of images and robot controllability are considered as good. The medical experts were able to recognize the internal structures of the echographic phantom and to follow them. The position errors of the slave robot are not very important for the medical expert because he controls the probe motion in function of what he sees in the echographic image -internal structures- rather than in function of the position of the real probe.

Next developments will consist in experimenting other control schemes, in particular hybrid position/force control which should allow to keep a more constant contact force with the body of the patient. In parallel, clinical validation will be performed ona larger number voluntary persons and on patients.

\section{Acknowledgments}

This project is supported by the French Ministry of Research and Technology (action line "ACI Telemédecine"), by France Telecom R\&D and by UAEM Mexico. We thank the clinicians, Dr Marc Althuser, Dr Jean Marc Ayoubi and M. Schneidern for testing the system. We also thanks PRAXIM, SINTERS, LVR and Pr Arbeille for their significant contribution to the project; and Dr. Kohji Masuda for lending us his vascular echographic phantom.

\section{References}

1. De Cunha, D., P. Gravez, C. Leroy, E. Maillard, J. Jouan, P. Varley, M. Jones, M. Halliwell, D. Hawkes, P.N.T. Wells and L. Angelini (1998). The midstep system for ultrasound guided remote telesurgery. 20th Annual International Conference of the IEEE Engineering in Medicine and Biology Society. Vol. 20. pp. 1266-1269. 
2. Gourdon, Ph. Poignet, G. Poisson, Y. Parmantier and P. Marché (1999)). Master-slave robotic system for ultrasound scanning. European Medical and Biological Engineering Conference. Vol. II. pp. 1116-1117.

3. S. Salcudean, G. Bell, S. Bachmann, W.H. Zhu, P Abolmaesumi and P.D. Lawrence (1999). Robot-assisted diagnostic ultrasound-design and feasibility experiments. Lecture Notes in Computer Science. Medical Image Computing and Computer-Assisted Intervention (MICCAI'99). pp. 1062-1071.

4. F. Pierrot, E. Dombre, E. Dégoulange, L. Urbain, P. Caron, S. Boudet, J. Gariépy and JL. Mégnien (1999). Hippocrate : a safe robot arm for medical applications with force feedback. Medical Image Analysis, Vol 3, pp 285-300.

5. on line http://www.crcg.edu/projects/teleinvivo.html.

6. M. Mitsuishi, S. Warisawa, T. Tsuda, T. Higuchi, N. Koizumi, H. Hashizume and K. Fujiwara. Remote Ultrasound Diagnostic System. Proceedings of the IEEE International conference on Robotics \& Automation, Seoul, Korea May 21-26 2001, pp 1567-1574.

7. R. P. Goldberg, M Dumitru, R. H. Taylor and D. Stoianovici. A Modular Robotic System for Ultrasound Image Acquisition. Fourth International Conference on Medical Image Computing and Computer Assisted Intervention, Utrecht, the Netherlands 14-17 October 2001, pp 1430-1432.

8. K. Masuda, E. Kimura, N. Tateishi and K. Ishihara. Three dimensional motion mechanism of ultrasound probe and its application for tele-echography system. Proceedings of the IEEE/RSJ International Conference on Intelligent Robots and Systems. Maui, Hawaii, USA, Oct 29-Nov 03 2001. pp 1112-1116.

9. Vilchis, P. Cinquin, J. Troccaz, A. Guerraz, B. Hennion, Franck Pellisier, Pierre Thorel, F. Courreges, Alain Gourdon, G. Poisson, Pierre Vieyres, Pierre Caron, Olivier Mérigeaux, Loïc Urbain, Cédric Daimo, Stéphan Lavallée, Philippe Arbeille, Marc Althuser, Jean Marc Ayoubi, B. Tondu and Serge Ippolito, "TER: a system for Robotic Tele-echography". Fourth International Conference on Medical Image Computing and Computer Assisted Intervention, Utrecht, the Netherlands 14-17 October 2001, pp 326-334.

10. Guerraz, A. Vilchis, J. Troccaz, P. Cinquin, B. Hennion, Franck Pellisier and Pierre Thorel, "A Haptic Virtual Environment for tele-Echography". 10 $0^{\text {th }}$ Annual Medicine Meets Virtual Reality Conference, Newport California January 23-26 2002.

11. Guerraz, B.Hennion, P. Thorel, F. Pellissier, A. Vienne and I. Belghit, "A haptic command station for remote ultrasound examinations", Tenth Annual Symposium on Haptic Interfaces for Virtual Environment and Teleoperator Systems, Orlando, FL, on March 24-25, 2002 in conjunction with the IEEE Virtual Reality 2002 Conference

12. Tondu and P. Lopez, "Modeling and control of Mckibben artificial muscle robot actuators", IEEE Control Systems Magazine, vol. 20, no. 2 pp 15-38, 2000.

13. Vlchis, J. Troccaz, P. Cinquin, F. Courrèges, G. Poisson and B. Tondu, "Robotic TeleUltrasound System (TER): Slave Robot Control", 1st IFAC Conference on Telematics Application in Automation and Robotics, Weingarten, Alemania 24-26 2001, pp 95-100.

14. F. Courreges, G. Poisson, P. Vieyres, A. Vilchis, J. Troccaz and P. Cinquin, "Low level control of antagonist artificial pneumatic muscles for a tele-operated ultrasound robot: TER”. 12 International Symposium on Measurement and Control in Robotics, (Bourges June 20-22 2002). 